\title{
SOME OPERATIONS ON TYPE-2 INTUITIONISTIC FUZZY SETS
}

\author{
BUI CONG CUONG ${ }^{1}$, TONG HOANG ANH, BUI DUONG HAI \\ ${ }^{1}$ Institute of Information Technology, Vietnam Academy of Science and Technology
}

Tóm tắt. Trong những thập niên gần đây, một số mở rộng của khái niệm tập mờ được đề xuất. Tập mờ loại hai và tập mờ trực cảm là hai khái niệm mới đã thu hút được nhiều sự quan tâm của các nhà nghiên cứu vì sự phong phú của các ứng dụng. Bài báo giới thiệu một khái niệm mới - tập mờ trực cảm loại hai và chứng minh một số tính chất trên đó.

\begin{abstract}
In the last decades, there have been some extensions of fuzzy sets and their applications. Recently, type-2 fuzzy set and intuitionistic fuzzy set are two of them, drawing a great deal of scientist's attention because of their widespread range of applications. In this paper, we introduce a new concept - type-2 intuitionistic fuzzy set and propose some properties of their operations.
\end{abstract}

\section{INTRODUCTION}

Type-2 fuzzy sets - that is, fuzzy sets with fuzzy sets as truth values - seem destined to play an increasingly important role in applications. They were introduced by Zadeh [13], extending the notion of ordinary fuzzy sets. The Mendel's book [5] on Uncertain Rule-based Fuzzy Logic Systems and other researches [6, 7, 8, 11] are discussions of both theoretical and practical aspects of type-2 fuzzy sets.

In [1] Atanassov K. introduced the concept of intuitionistic fuzzy set characterized by a membership function and a non-membership function, which is a generalization of fuzzy set. In [1] Atanassov K. also defined some operators of IFSs. Recently, intuitionistic fuzzy set (IFS) theory have been applied to many different fields, such as decision making, medical diagnosis, pattern recognition.

In this paper, we introduce concept of type- 2 intuitionistic fuzzy sets. We define some basic operations and derive some their properties. The paper is organized as follow: Section 2 gives a briefly review some basic definitions of fuzzy sets, type-2 fuzzy sets and intuitionistic fuzzy sets. Section 3 is devoted to the new main definitions and some their properties. Section 4 is a first discussion of a subclass of the type-2 IFS.

\section{BASIC DEFINITION}

\subsection{Definition of fuzzy sets}

Let $T, S$ be nonempty sets. The $\operatorname{Map}(S, T)$ be the set of all function from $S$ into $T$. A

\footnotetext{
${ }^{*}$ This paper was supported by NAFOSTED grant 102.01- 2012.14
} 
fuzzy set $A$ of a set $S$ is a maping $A: S \rightarrow[0,1]$. The set $S$ has no operations on it. So operations on the set $\operatorname{Map}(S,[0,1])$ of all fuzzy subsets of $S$ come from operations on $[0,1]$. Common operations on $[0,1]$ of interest in fuzzy theory are $\vee, \wedge$, and ' given by

$$
x \wedge y=\min \{x, y\}, \quad x \vee y=\max \{x, y\}, \quad x^{\prime}=1-x
$$

The constant 0 and 1 are generally considered as part of the algebraic structure. So the algebra basic to fuzzy set theory is $[0,1], \vee, \wedge,{ }^{\prime}, 0,1$.

The corresponding operations on the set $\operatorname{Map}(S,[0,1])$ of all fuzzy subsets of $S$ are given pointwise by the following formulas

$$
(A \wedge B)(s)=A(s) \wedge B(s), \quad(A \vee B)(s)=A(s) \vee B(s), \quad A^{\prime}(s)=(A(s))^{\prime}
$$

and the two nullary operations are given by $1(s)=1$ and $0(s)=0$ for all $s \in S$.

We use the same symbols for the pointwise operations on the elements of $\operatorname{Map}(S,[0,1])$. There are many properties hold in the algebra $I=\left([0,1], \vee, \wedge,{ }^{\prime} .0 .1\right)$ (see [9, 11]).

Thus, $I$ is a bounded distributive lattice with an involution ' that satisfies De Morgan's laws and the Kleene inequality. Thus is, $I$ is a Kleene algebra. Thus $\operatorname{Map}(S,[0,1])$ is also a Kleene algebra. Basic knowledge of fuzzy sets has been presented in $[3,9]$.

\subsection{Definition of type-2 fuzzy sets}

Let $S$ be a universe of discourse, the a type-2 fuzzy set (T2 FS) is defined as following.

Definition 2.2.1. [5] A type-2 fuzzy set, denoted by $A$, is characterized by a type-2 membership function $\mu_{A}(x, u)$, where $x \in S$ and $u \in J_{x} \subseteq[0.1]$, i.e. $A=\left\{\left((x, u), \mu_{A}(x, u)\right) \mid \forall x \in\right.$ $\left.X, \forall u \in J_{x} \subseteq[0.1]\right\}$, in which $0 \leq \mu_{A}(x, u) \leq 1$. A can be express as $A=\int_{x \in X} \int_{u \in J_{x}} \mu_{A}(x, u) /(x, u)$, $J_{x} \subseteq[0,1]$

\subsection{Basic definition and some properties of IFS}

Let $Y$ be a universe of discourse, then a fuzzy set $A=\left\{\left\langle y, \mu_{A}(y)\right\rangle \mid y \in Y\right\}$ defined by Zadeh [13] is characterized by a membership function $\mu_{A}: Y \rightarrow[0.1]$, where $\mu_{A}(y)$ denotes the degree of membership of element $y$ to the set $A$.

Definition 2.3.1. [1] An intuitionistic fuzzy set (IFS) $A=\left\{\left\langle y, \mu_{A}(y), \nu_{A}(y)\right\rangle \mid y \in Y\right\}$ is characterized by a membership function $\mu_{A}: Y \rightarrow[0.1]$, and a non-membership function $\nu_{A}: Y \rightarrow[0,1]$ with the condition $0 \leq \mu_{A}(y)+\nu_{A}(y) \leq 1$ for all $y \in Y$, where the numbers $\mu_{A}(y)$ and $\nu_{A}(y)$ represent the degree of membership and the degree of non-membership of the element $y$ to the set $A$, respectively.

Definition 2.3.2. [1, 4] If $A$ and $B$ are two IFS of the set $Y$, then $A \subset B$ iff $\forall y \in Y, \mu_{A}(y) \leq \mu_{B}(y)$ and $\nu_{A} \geq \nu_{B}(y), A \supset B$ iff $B \subset A$, $A=B$ iff $\forall y \in Y, \mu_{A}(y)=\mu_{B}(y)$ and $\nu_{A}=\nu_{B}(y)$, $A \cap B=\left\{<y, \min \left(\mu_{A}(y), \mu_{B}(y)\right), \max \left(\nu_{A}(y), \nu_{B}(y)\right)>\mid y \in Y\right\}$, $A \cup B=\left\{<y, \max \left(\mu_{A}(y), \mu_{B}(y)\right), \min \left(\nu_{A}(y), \nu_{B}(y)\right)>\mid y \in Y\right\}$, 
Definition 2.3.3. [1, 4] If $A_{1}$ and $A_{2}$ are two intuitionistic fuzzy sets, then $\left.\overline{A_{1}}=\left\{<y, \nu_{A_{1}}(y), \mu_{A_{1}}(y)\right\rangle \mid y \in Y\right\}$ $A_{1}+A_{2}=\left\{<y, \mu_{A_{1}}(y)+\mu_{A_{2}}(y)-\mu_{A_{1}}(y) \cdot \mu_{A_{2}}(y), \nu_{A_{1}}(y) \cdot \nu_{A_{2}}(y)>\mid y \in Y\right\}$ $A_{1} \cdot A_{2}=\left\{<y, \mu_{A_{1}}(y) \cdot \mu_{A_{2}}(y), \nu_{A_{1}}(y)+\nu_{A_{2}}(y)-\nu_{A_{1}}(y) \cdot \nu_{A_{2}}(y)>\mid y \in Y\right\}$ $\lambda A_{1}=\left\{<y, 1-\left(1-\mu_{A_{1}}(y)\right)^{\lambda},\left(\nu_{A_{1}}(y)\right)^{\lambda}>\mid y \in Y\right\}$ $A_{1}^{\lambda}=\left\{<y,\left(\mu_{A_{1}}(y)\right)^{\lambda}, 1-\left(1-\nu_{A_{1}}(y)\right)^{\lambda}>\mid y \in Y\right\}$

\section{DEFINITION OF OPERATIONS ON TYPE-2 IFS}

Now we introduce the notion of a type-2 intuitionistic fuzzy set.

\subsection{Definition}

Definition 3.1.1. Let $S$ be a nonempty set. $A$ is a type-2 intuitionistic fuzzy set (T2IFS) of $S . A$ is defined by: $A: S \rightarrow \operatorname{Map}(D,[0,1]) \times \operatorname{Map}(D,[0,1])$, where $D=\{(u, v) \in[0,1] \times[0,1]: u+v \leq 1\}$.

For convenience in description, the binary operations between $f(u, v)$ and $g(u, v)$ in $\operatorname{Map}(D,[0,1])$ are written in the forms $(f \wedge g)=(f \wedge g)(u, v)=f(u, v) \wedge g(u, v),(f \vee g)=(f \vee g)(u, v)=$ $f(u, v) \vee g(u, v)$ and $(f, g)=(f(u, v), g(u, v))$.

Now we give the definitions of main operations in T2IFS theory

\subsubsection{The operation AND}

Definition 3.1.2. Let $\left(f_{1}, g_{1}\right)$ and $\left(f_{2}, g_{2}\right)$ be in $\operatorname{Map}(D,[0,1]) \times \operatorname{Map}(D,[0,1])$. We define the intersection operation denoted $\sqcap$ and it is defined by: $\left(f_{1}, g_{1}\right) \sqcap\left(f_{2}, g_{2}\right)=(f, g)$ where for any $(u, v) \in D$

$$
\begin{aligned}
& f(u, v)=\underset{u_{1} \wedge u_{2}=u, v_{1} \vee v_{2}=v}{\vee} f_{1}\left(u_{1}, v_{1}\right) \wedge f_{2}\left(u_{2}, v_{2}\right) \\
& \underset{u_{1} \wedge u_{2}=u, v_{1} \vee v_{2}=v}{V} g_{1}\left(u_{1}, v_{1}\right) \wedge g_{2}\left(u_{2}, v_{2}\right)
\end{aligned}
$$

\subsubsection{The operation OR}

Definition 3.1.3. Let $\left(f_{1}, g_{1}\right)$ and $\left(f_{2}, g_{2}\right)$ be in $\operatorname{Map}(D,[0,1]) \times \operatorname{Map}(D,[0,1])$. We define the union operation denoted $\sqcup$ and it is defined by: $\left(f_{1}, g_{1}\right) \sqcup\left(f_{2}, g_{2}\right)=(f, g)$ where for any $(u, v) \in D$

$$
\begin{aligned}
& f(u, v)=\underset{u_{1} \vee u_{2}=u, v_{1} \wedge v_{2}=v}{\vee} f_{1}\left(u_{1}, v_{1}\right) \wedge f_{2}\left(u_{2}, v_{2}\right), \\
& g(u, v)=\underset{u_{1} \vee u_{2}=u, v_{1} \wedge v_{2}=v}{\vee} g_{1}\left(u_{1}, v_{1}\right) \wedge g_{2}\left(u_{2}, v_{2}\right)
\end{aligned}
$$

\subsubsection{The operation NEGATION}

$$
(f(u, v), g(u, v))^{*}=(f(v, u), g(v, u))
$$

The followings are definitions of identities. They are $\mathbf{1}=\left(\mathbf{1}_{1}, \mathbf{1}_{0}\right)$ and $\mathbf{0}=\left(\mathbf{1}_{0}, \mathbf{1}_{1}\right)$. 


$$
\mathbf{1}_{1}(u, v)=\left\{\begin{array}{ll}
1 & \text { if } u=1, v=0 \\
0 & \text { if } u \neq 1, v \neq 0
\end{array} \mathbf{1}_{0}(u, v)= \begin{cases}1 & \text { if } u=0, v=1 \\
0 & \text { if } u \neq 0, v \neq 1\end{cases}\right.
$$

Thus, we defined the algebra $\mathbf{M}=\left(\operatorname{Map}(D,[0,1]) \times \operatorname{Map}(D,[0,1]), \sqcap, \sqcup,{ }^{*}, \mathbf{1}, \mathbf{0}\right)$ for T2IFS with operations $\sqcap, \sqcup,{ }^{*}$. Our aim in this paper is to examine some properties on the algebra of T2IFS such as idempotent, involution, commutative laws, associative laws or distributive laws.

\subsection{Some properties of these operations.}

In this section, we are going to demonstrate some properties of the operations on T2 IFS. We start with the below theorem which clarifies the first properties such as idempotent, commutative, absorption laws with identities, involution, and De Morgan's laws.

Theorem 3.2.1. For every $(f, g),\left(f_{1}, g_{1}\right),\left(f_{2}, g_{2}\right) \in \boldsymbol{M}$, we have

1. $(f, g) \sqcap(f, g)=(f, g)$ and $(f, g) \sqcup(f, g)=(f, g)$

2. $\left(f_{1}, g_{1}\right) \sqcap\left(f_{2}, g_{2}\right)=\left(f_{2}, g_{2}\right) \sqcap\left(f_{1}, g_{1}\right)$ and $\left(f_{1}, g_{1}\right) \sqcup\left(f_{2}, g_{2}\right)=\left(f_{2}, g_{2}\right) \sqcup\left(f_{1}, g_{1}\right)$

3. $\left(\mathbf{1}_{1}, \mathbf{1}_{0}\right) \sqcap(f, g)=(f, g)$ and $\left(\mathbf{1}_{0}, \mathbf{1}_{1}\right) \sqcup(f, g)=(f, g)$

4. $(f, g)^{* *}=(f, g)$

5. $\left\{\left(f_{1}, g_{1}\right) \sqcap\left(f_{2}, g_{2}\right)\right\}^{*}=\left(f_{1}, g_{1}\right)^{*} \sqcup\left(f_{2}, g_{2}\right)^{*}$ and

$\left\{\left(f_{1}, g_{1}\right) \sqcup\left(f_{2}, g_{2}\right)\right\}^{*}=\left(f_{1}, g_{1}\right)^{*} \sqcap\left(f_{2}, g_{2}\right)^{*}$

Proof We omit properties 1,2 and 4 .

The proof of property 3 . First, we handle the absorption law of identity $\left(\mathbf{1}_{1}, \mathbf{1}_{0}\right)$. Let $(f, g)$ be in M.Suppose that $\left(\mathbf{1}_{1}, \mathbf{1}_{0}\right) \sqcap(f, g)=\left(f^{\prime}, g^{\prime}\right)$, we have

$$
f^{\prime}(u, v)=\underset{u_{1} \wedge u_{2}=u, v_{1} \vee v_{2}=v}{\vee} \mathbf{1}_{1}\left(u_{1}, v_{1}\right) \wedge f\left(u_{2}, v_{2}\right)
$$

To find $f^{\prime}(u, v)$ we look for all values of $\mathbf{1}_{1}\left(u_{1}, v_{1}\right) \wedge f\left(u_{2}, v_{2}\right)$, where $u_{1} \wedge u_{2}=u, v_{1} \vee v_{2}=v$ and then find their sup. In the first case, if $\left(u_{1}, v_{1}\right)=(1,0)$ then $\mathbf{1}_{1}\left(u_{1}, v_{1}\right)=1$ and $\left(u_{2}, v_{2}\right)$ must be $(u, v)$. We have $\mathbf{1}_{1}\left(u_{1}, v_{1}\right) \wedge f\left(u_{2}, v_{2}\right)=1 \wedge f(u, v)=f(u, v)$.

In the other case, if $\left(u_{1}, v_{1}\right) \neq(1,0)$, then $\mathbf{1}_{1}\left(u_{1}, v_{1}\right)=0$, we have $\mathbf{1}_{1}\left(u_{1}, v_{1}\right) \wedge f\left(u_{2}, v_{2}\right)=$ $0 \wedge f\left(u_{2}, v_{2}\right)=0$.

Hence, $f^{\prime}(u, v)$ is the sup of two results for the two cases or $f^{\prime}(u, v)=0 \vee f(u, v)=f(u, v)$. In similar way, we prove that $g^{\prime}(u, v)=g(u, v)$.

With the same arguments, the absorption law of the other identity can be proven.

For property 5,we prove only the first formula. The second formula automatically has the analogous proof.

Let $\left(f_{1}, g_{1}\right),\left(f_{2}, g_{2}\right) \in \mathbf{M}$. Suppose that $\left(f^{\prime}, g^{\prime}\right)=\left(f_{1}, g_{1}\right) \sqcap\left(f_{2}, g_{2}\right)$, we have

$$
\begin{aligned}
f^{\prime}(u, v) & =\underset{u_{1} \wedge u_{2}=u, v_{1} \vee v_{2}=v}{\vee} f_{1}\left(u_{1}, v_{1}\right) \wedge f_{2}\left(u_{2}, v_{2}\right), \\
g^{\prime}(u, v) & =\underset{u_{1} \wedge u_{2}=u, v_{1} \vee v_{2}=v}{\vee} g_{1}\left(u_{1}, v_{1}\right) \wedge g_{2}\left(u_{2}, v_{2}\right) .
\end{aligned}
$$

then $\left(f^{\prime}(u, v), g^{\prime}(u, v)\right)^{*}=\left(f^{\prime}(v, u), g^{\prime}(v, u)\right)$, where

$$
\begin{aligned}
f^{\prime}(v, u) & =\underset{u_{1} \wedge u_{2}=u, v_{1} \vee v_{2}=v}{\vee} f_{1}\left(v_{1}, u_{1}\right) \wedge f_{2}\left(v_{2}, u_{2}\right), \\
g^{\prime}(v, u) & =\underset{u_{1} \wedge u_{2}=u, v_{1} \vee v_{2}=v}{\vee} g_{1}\left(v_{1}, u_{1}\right) \wedge g_{2}\left(v_{2}, u_{2}\right) .
\end{aligned}
$$


Otherwise,

$\left(f_{1}, g_{1}\right)^{*} \sqcup\left(f_{2}, g_{2}\right)^{*}=\left(f_{1}(u, v), g_{1}(u, v)\right)^{*} \sqcup\left(f_{2}(u, v), g_{2}(u, v)\right)^{*}$ $=\left(f_{1}(v, u), g_{1}(v, u)\right) \sqcup\left(f_{2}(v, u), g_{2}(v, u)=\left(f^{\prime \prime}(v, u), g^{\prime \prime}(v, u)\right)\right.$, where

$$
\begin{aligned}
& f^{\prime \prime}(v, u)=\underset{v_{1} \vee v_{2}=v, u_{1} \wedge u_{2}=u}{\vee} f_{1}\left(v_{1}, u_{1}\right) \wedge f_{2}\left(v_{2}, u_{2}\right), \\
& g^{\prime \prime}(v, u)=\underset{v_{1} \vee v_{2}=v, u_{1} \wedge u_{2}=u}{\vee} g_{1}\left(v_{1}, u_{1}\right) \wedge g_{2}\left(v_{2}, u_{2}\right) .
\end{aligned}
$$

Comparing $\left(f^{\prime}, g^{\prime}\right)$ and $\left(f^{\prime \prime}, g^{\prime \prime}\right)$, we can imply $\left(f^{\prime}, g^{\prime}\right)=\left(f^{\prime \prime}, g^{\prime \prime}\right)$. Thus, we have $\left\{\left(f_{1}, g_{1}\right) \sqcap\right.$ $\left.\left(f_{2}, g_{2}\right)\right\}^{*}=\left(f_{1}, g_{1}\right)^{*} \sqcup\left(f_{2}, g_{2}\right)^{*}$ is proved.

Definition 3.2.2. For $f \in \operatorname{Map}(D,[0,1])$. Let $f^{L}, f^{R}, f_{L}$ and $f_{R}$ be elements of $\operatorname{Map}(D,[0,1])$ defined by $f^{L}(u, v)=\vee_{u^{\prime} \leq u} f\left(u^{\prime}, v\right), f^{R}(u, v)=\vee_{u^{\prime} \geq u} f\left(u^{\prime}, v\right), f_{L}(u, v)=\vee_{v^{\prime} \leq v} f\left(u, v^{\prime}\right)$, $f_{R}(u, v)=\vee_{v^{\prime} \geq v} f\left(u, v^{\prime}\right)$.

The below figures visualize our definitions.

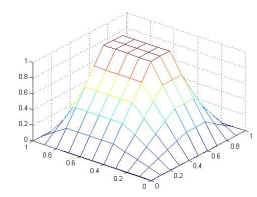

(a) $f$

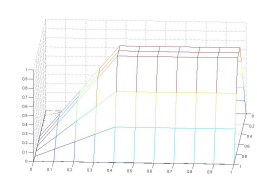

(b) $f^{L}$

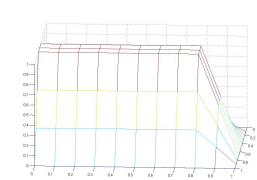

(c) $f^{R}$

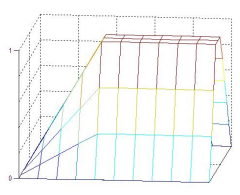

(d) $f_{L}$

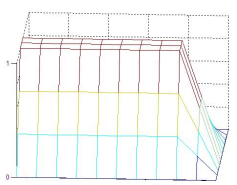

(e) $f_{R}$

Figure 3.1: Geometrical interpretation of $f, f^{L}, f^{R}, f_{L}$, and $f_{R}$

Theorem 3.2.3. The following properties hold for all $\left(f_{1}, g_{1}\right)\left(f_{2}, g_{2}\right) \in \boldsymbol{M}$ :

1. $\left(f_{1}, g_{1}\right) \sqcap\left(f_{2}, g_{2}\right)=(f, g)$ provided

$$
\begin{aligned}
& f=\left(f_{1_{L}} \wedge f_{2}^{R}\right) \vee\left(f_{1}^{R} \wedge f_{2_{L}}\right) \vee\left(f_{1 L}^{R} \wedge f_{2}\right) \vee\left(f_{1} \wedge f_{2}^{R}\right) \\
& g=\left(g_{1 L} \wedge g_{2}^{R}\right) \vee\left(g_{1}^{R} \wedge g_{2 L}\right) \vee\left(g_{1}^{R} \wedge g_{2}\right) \vee\left(g_{1} \wedge g_{2}^{R}\right)
\end{aligned}
$$

2. $\left(f_{1}, g_{1}\right) \sqcup\left(f_{2}, g_{2}\right)=(f, g)$ provided

$$
\begin{aligned}
& f=\left(f_{1}{ }^{L} \wedge f_{2_{R}}\right) \vee\left(f_{1 R} \wedge f_{2}{ }^{L}\right) \vee\left(f_{1}{ }_{R} \wedge f_{2}\right) \vee\left(f_{1} \wedge f_{2}{ }_{R}^{L}\right) \\
& g=\left(g_{1}{ }^{L} \wedge g_{2}\right) \vee\left(g_{1} \wedge g_{2}{ }^{L}\right) \vee\left(g_{1} L \wedge g_{2}\right) \vee\left(g_{1} \wedge g_{2}{ }_{R}\right)
\end{aligned}
$$

\section{Proof}

Let $\left(f_{1}, g_{1}\right),\left(f_{2}, g_{2}\right) \in \mathbf{M}$. We have $\left(f_{1}, g_{1}\right) \sqcap\left(f_{2}, g_{2}\right)=(f, g)$ such that

$$
\begin{aligned}
& f(u, v)=\bigvee_{u_{1} \wedge u_{2}=u, v_{1} \vee v_{2}=v}\left(f_{1}\left(u_{1}, v_{1}\right) \wedge f_{2}\left(u_{2}, v_{2}\right)\right), \\
& g(u, v)=\bigvee_{u_{1} \wedge u_{2}=u, v_{1} \vee v_{2}=v}\left(g_{1}\left(u_{1}, v_{1}\right) \wedge g_{2}\left(u_{2}, v_{2}\right)\right)
\end{aligned}
$$


First,

$$
\begin{aligned}
& f(u, v)=\bigvee_{u_{1} \wedge u_{2}=u, v_{1} \vee v_{2}=v}\left(f_{1}\left(u_{1}, v_{1}\right) \wedge f_{2}\left(u_{2}, v_{2}\right)\right) \\
& =\bigvee_{u_{1} \wedge u_{2}=u}^{u_{1}}\left[\bigvee_{v_{1} \vee v_{2}=v}\left(f_{1}\left(u_{1}, v_{1}\right) \wedge f_{2}\left(u_{2}, v_{2}\right)\right)\right] \\
& =\bigvee_{u_{1} \wedge u_{2}=u}^{u_{1} \wedge u_{2}=u}\left\{\left[\bigvee_{v_{1} \vee v=v}^{v_{1} \vee v_{2}=v}\left(f_{1}\left(u_{1}, v_{1}\right) \wedge f_{2}\left(u_{2}, v\right)\right)\right] \vee\left[\bigvee_{v \vee v_{2}=v}\left(f_{1}\left(u_{1}\right) \wedge f_{2}\left(u_{2}, v_{2}\right)\right)\right]\right\} \\
& =\bigvee_{u_{1} \wedge u_{2}=u}^{u_{1} \wedge u_{2}=u}\left\{\left[\left(\bigvee_{v_{1} \leq v}^{v_{1} \vee v=v} f_{1}\left(u_{1}, v_{1}\right)\right) \wedge f_{2}\left(u_{2}, v\right)\right] \vee\left[f_{1}\left(u_{1}, v\right) \wedge\left(\bigvee_{v 2 \leq v} f_{2}\left(u_{2}, v_{2}\right)\right)\right]\right\} \\
& =\bigvee_{u_{1} \wedge u_{2}=u}\left(\left(f_{1 L}\left(u_{1}, v\right) \wedge f_{2}\left(u_{2}, v\right)\right) \vee\left(f_{1}\left(u_{1}, v\right) \wedge f_{2_{L}}\left(u_{2}, v\right)\right)\right) \\
& =\bigvee_{u_{1} \wedge u_{2}=u}\left(f_{1 L}\left(u_{1}, v\right) \wedge f_{2}\left(u_{2}, v\right)\right) \vee \bigvee_{u_{1} \wedge u_{2}=u}\left(f_{1}\left(u_{1}, v\right) \wedge f_{2 L}\left(u_{2}, v\right)\right) \\
& =\bigvee_{u \wedge u_{2}=u}^{u_{1} \wedge u_{2}=u}\left(f_{1 L}(u, v) \wedge f_{2}\left(u_{2}, v\right)\right) \vee \bigvee_{u_{1} \wedge u=u}^{u_{1} \wedge u_{2}=u}\left(f_{1 L}\left(u_{1}, v\right) \wedge f_{2}(u, v)\right) \\
& \vee \bigvee_{u \wedge u_{2}=u}\left(f_{1}(u, v) \wedge f_{2_{L}}\left(u_{2}, v\right)\right) \vee \bigvee_{u_{1} \wedge u=u}\left(f_{1}\left(u_{1}, v\right) \wedge f_{2_{L}}(u, v)\right) \\
& =\left(f_{1_{L}}(u, v) \wedge \bigvee_{u_{2} \geq u} f_{2}\left(u_{2}, v\right)\right) \vee\left(\bigvee_{u_{1} \geq u} f_{1_{L}}\left(u_{1}, v\right) \wedge f_{2}(u, v)\right) \\
& \vee\left(f_{1}(u, v) \wedge \bigvee_{u_{2} \geq u}^{u_{2} \geq u} f_{2 L}\left(u_{2}, v\right)\right) \vee\left(\bigvee_{u_{1} \geq u}^{u_{1} \geq u} f_{1}\left(u_{1}, v\right) \wedge f_{2 L}(u, v)\right) \\
& =\left(f_{1 L}(u, v) \wedge f_{2}^{R}(u, v)\right) \vee\left(f_{1}^{R}\left(u_{1}^{R}, v\right) \wedge f_{2}(u, v)\right) \vee\left(f_{1}(u, v) \wedge f_{2}^{R}(u, v)\right) \\
& \vee\left(f_{1}^{R}(u, v) \wedge f_{2 L}(u, v)\right) \\
& =\left(f_{1 L} \wedge f_{2}^{R}\right) \vee\left(f_{1}^{R} \wedge f_{2 L}\right) \vee\left(f_{1}^{R} \wedge f_{2}\right) \vee\left(f_{1} \wedge f_{2}^{R}\right)
\end{aligned}
$$

we can easily prove the fomulas stated for $g$. It is no doubt that the operation $\sqcup$ between $\left(f_{1}, g_{1}\right)$ and $\left(f_{2}, g_{2}\right)$ has the similar proof.

Note.To make convenient for further descriptions and proofs, let denote operations between $\left(f_{1}, g_{1}\right)$ and $\left(f_{2}, g_{2}\right)$ as the followings:

1. $\left(f_{1}, g_{1}\right) \sqcup\left(f_{2}, g_{2}\right)=\left(f_{1} \uplus f_{2}, g_{1} \uplus g_{2}\right)$, where

$f_{1} \cup f_{2}=\left(f_{1}^{L} \wedge f_{2 R}\right) \vee\left(f_{1} \wedge f_{2}^{L}\right) \vee\left(f_{1}^{L} \wedge f_{2}\right) \vee\left(f_{1} \wedge f_{2}^{L}\right)$

$g_{1}$ ש $g_{2}=\left(g_{1} L \wedge g_{2}\right) \vee\left(g_{1} \wedge g_{2} L\right) \vee\left(g_{1}^{L} \wedge g_{2}\right) \vee\left(g_{1} \wedge g_{2} L\right)$

$\left(f_{1}, g_{1}\right) \sqcap\left(f_{2}, g_{2}\right)=\left(f_{1} \cap f_{2}, g_{1} \cap g_{2}\right)$ where

$f_{1}$ ก $f_{2}=\left(f_{1} \wedge f_{2}{ }^{R}\right) \vee\left(f_{1}^{R} \wedge f_{2 L}\right) \vee\left(f_{1}^{R} \wedge f_{2}\right) \vee\left(f_{1} \wedge f_{2 L}^{R}\right)$

$g_{1}$ ก $g_{2}=\left(g_{1} \wedge g_{2}{ }^{R}\right) \vee\left(g_{1}^{R} \wedge g_{2}\right) \vee\left(g_{1}^{R} \wedge g_{2}\right) \vee\left(g_{1} \wedge g_{2}^{R}\right)$

2.f $\cap$ ก $g=\left(f_{L} \nabla g\right) \vee\left(f \nabla g_{L}\right)$ and $f \in g=\left(f_{R} \triangle g\right) \vee\left(f \triangle g_{R}\right)$

such that

$f \nabla g=\left(f \wedge g^{R}\right) \vee\left(f^{R} \wedge g\right)$ and $f \triangle g=\left(f \wedge g^{L}\right) \vee\left(f^{L} \wedge g\right)$

Corollary 3.2.4. Let $f, g \in \operatorname{Map}(D,[0,1])$. We have

1. $(f \uplus g)^{L}=\left(f_{R}^{L} \wedge g^{L}\right) \vee\left(f^{L} \wedge g_{R}^{L}\right)$ and $(f \uplus g)_{R}=\left(f_{R}^{L} \wedge g_{R}\right) \vee\left(f_{R} \wedge g_{R}^{L}\right)$.

2. $(f \cap g)_{R}=\left(f_{R L} \nabla g_{R}\right) \vee\left(f_{R} \nabla g_{R L}\right)$ and $(f \uplus g)_{L}=\left(f_{R L} \triangle g_{L}\right) \vee\left(f_{L} \triangle g_{R L}\right)$.

3. $(f \uplus g)_{R}^{L}=f_{R}^{L} \wedge g_{R}^{L}$.

\section{Proof}

1.We have 


$$
\begin{aligned}
& (f \uplus g)^{L}(u, v)=\bigvee_{u^{\prime} \leq u}(f \uplus g)\left(u^{\prime}, v\right)=\bigvee_{u^{\prime} \leq u}\left(\bigvee_{u_{1} \vee u_{2}=u^{\prime}, v_{1} \wedge v_{2}=v}\left(f\left(u_{1}, v_{1}\right) \wedge g\left(u_{2}, v_{2}\right)\right)\right) \\
& =\bigvee_{u_{1} \vee u_{2} \leq u}\left(\bigvee_{v_{1} \wedge v_{2}=v}\left(f\left(u_{1}, v_{1}\right) \wedge g\left(u_{2}, v_{2}\right)\right)\right) \\
& =\bigvee_{u_{1} \vee u_{2} \leq u}\left(\left(f_{R}\left(u_{1}, v\right) \wedge g\left(u_{2}, v\right)\right) \vee\left(f\left(u_{1}, v\right) \wedge g_{R}\left(u_{2}, v\right)\right)\right) \\
& =\underset{u_{1} \vee u_{2} \leq u}{\bigvee}\left(f_{R}\left(u_{1}, v\right) \wedge g\left(u_{2}, v\right)\right) \vee \bigvee_{u_{1} \vee u_{2} \leq u}\left(f\left(u_{1}, v\right) \wedge g_{R}\left(u_{2}, v\right)\right) \quad \text { There is } \\
& =\bigvee_{u_{1} \leq u, u_{2} \leq u}^{u_{1} \vee u_{2} \leq u}\left(f_{R}\left(u_{1}, v\right) \wedge g\left(u_{2}, v\right)\right) \vee \bigvee_{u_{1} \leq u, u_{2} \leq u}^{u_{1} \vee u_{2} \leq u}\left(f\left(u_{1}, v\right) \wedge g_{R}\left(u_{2}, v\right)\right) \\
& =\left(\bigvee_{u_{1}<u} f_{R}\left(u_{1}, v\right) \wedge \bigvee_{u_{2} \leq u} g\left(u_{2}, v\right)\right) \vee\left(\bigvee_{u_{1}<u} f\left(u_{1}, v\right) \wedge \bigvee_{u_{2}<u} g_{R}\left(u_{2}, v\right)\right) \\
& =\left(f_{R}^{L}(u, v) \wedge g^{L}(u, v)\right) \vee\left(f^{L}(u, v) \wedge g_{R}^{L}(u, v)\right) \text {. }
\end{aligned}
$$

no difficulty to prove the other formula by using the same arguments used in the above proof.

2. We have

$$
\begin{aligned}
(f \cap g)_{R}(u, v) & =\bigvee_{v^{\prime} \geq v}(f \cap g)\left(u, v^{\prime}\right)=\bigvee_{v^{\prime} \geq v} \bigvee_{u_{1} \wedge u_{2}=u, v_{1} \vee v_{2}=v^{\prime}} f\left(u_{1}, v_{1}\right) \wedge g\left(u_{2}, v_{2}\right) \\
& =\bigvee_{u_{1} \wedge u_{2}=u, v_{1} \vee v_{2} \geq v} f\left(u_{1}, v_{1}\right) \wedge g\left(u_{2}, v_{2}\right) \\
& =\bigvee_{v_{1} \vee v_{2} \geq v} \bigvee_{u u_{1} \wedge u_{2}=u} f\left(u_{1}, v_{1}\right) \wedge g\left(u_{2}, v_{2}\right) \\
& =\bigvee_{v_{1} \vee v_{2} \geq v}\left[\left(\bigvee_{u \wedge u_{2}=u} f\left(u, v_{1}\right) \wedge g\left(u_{2}, v_{2}\right)\right) \vee\left(\bigvee_{u_{1} \wedge u=u} f\left(u_{1}, v_{1}\right) \wedge g\left(u, v_{2}\right)\right)\right] \\
& =\bigvee_{v_{1} \vee v_{2} \geq v}\left[\left(f\left(u, v_{1}\right) \wedge \bigvee_{u_{2} \geq u} g\left(u_{2}, v_{2}\right)\right) \vee\left(\left(\bigvee_{u_{1} \geq u} f\left(u_{1}, v_{1}\right)\right) \wedge g\left(u, v_{2}\right)\right)\right] \\
& =\bigvee_{v_{1} \vee v_{2} \geq v}\left[\left(f\left(u, v_{1}\right) \wedge g^{R}\left(u, v_{2}\right)\right) \vee\left(f^{R}\left(u, v_{1}\right) \wedge g\left(u, v_{2}\right)\right)\right] \\
& =\bigvee_{v_{1} \geq v, v_{2}}\left[\left(f\left(u, v_{1}\right) \wedge g^{R}\left(u, v_{2}\right)\right) \vee\left(f^{R}\left(u, v_{1}\right) \wedge g\left(u, v_{2}\right)\right)\right] \vee \\
& v_{2} \geq v, v_{1} \\
& =\left[\left(f\left(u, v_{1}\right) \wedge g^{R}(u, v) \wedge g_{R L}^{R}(u, v)\right) \vee\left(f_{2}\right) \vee\left(f^{R}\left(u, v_{1}\right) \wedge g\left(u, v_{2}\right)\right)\right] \\
& {\left[\left(f_{R L}(u, v) \wedge g_{R}^{R}(u, v)\right) \vee\left(f_{R L}^{R}(u, v) \wedge g_{R L}(u, v)\right)\right] \vee } \\
& =\left(f_{R} \wedge g_{R L}^{R}\right) \vee\left(f_{R}^{R} \wedge g_{R L}\right) \vee\left(f_{R L} \wedge g_{R}^{R}\right) \vee\left(f_{R L}^{R} \wedge g_{R}\right) \\
& =\left(f_{R} \nabla g_{R L}\right) \vee\left(f_{R L} \nabla g_{R}\right) .
\end{aligned}
$$

$(f \cap g)_{R}=\left(f_{R L} \nabla g_{R}\right) \vee\left(f_{R} \nabla g_{R L}\right)$.

With the same arguments, it becomes easy to prove the following formula:

$$
(f \uplus g)_{L}=\left(f_{R L} \triangle g_{L}\right) \vee\left(f_{L} \triangle g_{R L}\right) .
$$

3. We have

$$
\begin{aligned}
& \begin{array}{l}
\text { 3. We have } \\
(f \uplus)_{L}^{R}
\end{array}=\bigvee_{u^{\prime} \leq u, v^{\prime} \geq v}(f \uplus g)\left(u^{\prime}, v^{\prime}\right)=\bigvee_{u^{\prime} \leq u, v^{\prime} \geq v}\left(\underset{u_{1} \vee u_{2}=u^{\prime}, v_{1} \wedge v_{2}=v^{\prime}}{\bigvee}\left(f\left(u_{1}, v_{1}\right) \wedge g\left(u_{2}, v_{2}\right)\right)\right) \\
& =\bigvee_{u_{1} \vee u_{2} \leq u, v_{1} \wedge v_{2}>v}\left(f\left(u_{1}, v_{1}\right) \wedge g\left(u_{2}, v_{2}\right)=\bigvee_{u_{1} \vee u_{2} \leq u}\left(\bigvee_{v_{1} \wedge v_{2}>v}\left(f\left(u_{1}, v_{1}\right) \wedge g\left(u_{2}, v_{2}\right)\right)\right.\right. \\
& =\bigvee_{u_{1} \vee u_{2} \leq u}\left(\bigvee_{v_{1} \geq v} f\left(u_{1}, v_{1}\right) \wedge \bigvee_{v_{2} \geq v} g\left(u_{2}, v_{2}\right)=\bigvee_{u_{1} \vee u_{2} \leq u}\left(f_{R}\left(u_{1}, v\right) \wedge g_{R}\left(u_{2}, v\right)\right.\right. \\
& =\left(\bigvee_{u_{1} \leq u}\left(f_{R}\left(u_{1}, v\right)\right) \wedge\left(\bigvee_{u_{2} \leq u} g_{R}\left(u_{2}, v\right)\right)=f_{R}^{L}(u, v) \wedge g_{R}^{L}(u, v)\right. \\
& =f_{R}^{L} \wedge g_{R}^{L}
\end{aligned}
$$

Theorem 3.2.5. The following associative laws hold for $\sqcup$ and $\sqcap$.

$$
\left(f_{1}, f_{2}\right) \sqcup\left[\left(g_{1}, g_{2}\right) \sqcup\left(h_{1}, h_{2}\right)\right]=\left[\left(f_{1}, f_{2}\right) \sqcup\left(g_{1}, g_{2}\right)\right] \sqcup\left(h_{1}, h_{2}\right)
$$




$$
\left(f_{1}, f_{2}\right) \sqcap\left[\left(g_{1}, g_{2}\right) \sqcap\left(h_{1}, h_{2}\right)\right]=\left[\left(f_{1}, f_{2}\right) \sqcap\left(g_{1}, g_{2}\right)\right] \sqcap\left(h_{1}, h_{2}\right)
$$

\section{Proof}

We are going to prove the following formula. For $f, g, h \in \operatorname{Map}(D,[0,1])$

$$
f \uplus(g \uplus h)=(f \uplus g) \uplus h
$$

From the right side of (1), we have

$$
\begin{aligned}
(f \uplus g) \uplus h & =\left[(f \uplus g)^{L} \wedge h_{R}\right] \vee\left[(f \uplus g)_{R} \wedge h^{L}\right] \vee\left[(f \cup g)_{R}^{L} \wedge h\right] \vee\left[(f \uplus g) \wedge h_{R}^{L}\right] \\
& =\left\{\left[\left(f_{R}^{L} \wedge g^{L}\right) \vee\left(f \wedge g_{R}^{L}\right)\right] \wedge h_{R}\right\} \vee\left\{\left[\left(f g_{R}^{L} \wedge g_{R}\right) \vee\left(f_{R} \wedge g_{R}^{L}\right)\right] \wedge h^{L}\right\} \\
& \vee\left[\left(f_{R}^{L} \wedge g_{R}^{L}\right) \wedge h\right] \vee\left\{\left[\left(f^{L} \wedge g_{R}\right) \vee\left(f_{R} \wedge g^{L}\right) \vee\left(f_{R}^{L} \wedge g\right) \vee\left(f \wedge g_{R}^{L}\right)\right] \wedge h_{R}^{L}\right\} \quad \text { From } \\
& =\left[f_{R}^{L} \wedge g^{L} \wedge h_{R}\right] \vee\left[f^{L} \wedge g_{R}^{L} \wedge h_{R}\right] \vee\left[f_{R}^{L} \wedge g_{R} \wedge h^{L}\right] \vee\left[f_{R} \wedge g_{R}^{L} \wedge h^{L}\right] \\
& \vee\left[f_{R}^{L} \wedge g_{R}^{L} \wedge h\right] \vee\left[f^{L} \wedge g_{R} \wedge h_{R}^{L}\right] \vee\left[f_{R} \wedge g^{L} \wedge h_{R}^{L}\right] \vee\left[f_{R}^{L} \wedge g \wedge h_{R}^{L}\right] \\
& \vee\left[f \wedge g_{R}^{L} \wedge h_{R}^{L}\right]
\end{aligned}
$$

the left side of $(1)$, we have

$$
\begin{aligned}
f \uplus(g \uplus h) & =\left[f^{L} \wedge(g \uplus h)_{R}\right] \vee\left[f_{R} \wedge(g \uplus h)^{L}\right] \vee\left[f_{R}^{L} \wedge(g \uplus h)\right] \vee\left[f \wedge(g \uplus h)_{R}^{L}\right] \\
& =\left\{f^{L} \wedge\left[\left(g_{R}^{L} \wedge h_{R}\right) \vee\left(g_{R} \wedge h_{R}^{L}\right)\right]\right\} \vee\left\{f_{R} \wedge\left[\left(g_{R}^{L} \wedge h^{L}\right) \vee\left(g^{L} \wedge h_{R}^{L}\right)\right]\right\} \\
& \vee\left\{f_{R}^{L} \wedge\left[\left(g^{L} \wedge h_{R}\right) \vee\left(g_{R} \wedge h^{L}\right) \vee\left(g_{R}^{L} \wedge h\right) \vee\left(g \wedge h_{R}^{L}\right)\right]\right\} \vee\left[f \wedge\left(g_{R}^{L} \wedge h_{R}^{L}\right)\right] \\
& =\left[f^{L} \wedge g_{R}^{L} \wedge h_{R}\right] \vee\left[f^{L} \wedge g_{R} \wedge h_{R}^{L}\right] \vee\left[f_{R} \wedge g_{R}^{L} \wedge h^{L}\right] \vee\left[f_{R} \wedge g^{L} \wedge h_{R}^{L}\right] \\
& \vee\left[f_{R}^{L} \wedge g^{L} \wedge h_{R}\right] \vee\left[f_{R}^{L} \wedge g_{R} \wedge h^{L}\right] \vee\left[f_{R}^{L} \wedge g_{R}^{L} \wedge h\right] \vee\left[f_{R}^{L} \wedge g \wedge h_{R}^{L}\right] \\
& \vee\left[f \wedge g_{R}^{L} \wedge h_{R}^{L}\right]
\end{aligned}
$$

Hence, $f \uplus(g \uplus h)=(f \uplus g) \cup h$ is proved. Because of this property, we imply

$$
\begin{aligned}
\left(f_{1}, f_{2}\right) \sqcup\left[\left(g_{1}, g_{2}\right) \sqcup\left(h_{1}, h_{2}\right)\right] & =\left(f_{1} \uplus\left(g_{1} \uplus h_{1}\right), f_{2} \uplus\left(g_{2} \uplus h_{2}\right)\right) \\
& =\left(\left(f_{1} \uplus g_{1}\right) \uplus h_{1},\left(f_{2} \uplus g_{2}\right) \uplus h_{2}\right) \\
& =\left[\left(f_{1}, f_{2}\right) \sqcup\left(g_{1}, g_{2}\right)\right] \sqcup\left(h_{1}, h_{2}\right)
\end{aligned}
$$

To prove the absorption law of $\sqcap$, we have the similar proof.

Corollary 3.2.6. The following distributive laws hold for operations $\nabla, \triangle$ and $\vee$ :

$$
\begin{aligned}
& f \nabla(g \vee h)=(f \nabla g) \vee(f \nabla h) \\
& f \triangle(g \vee h)=(f \triangle g) \vee(f \triangle h)
\end{aligned}
$$

This is one of the results which has been proved for type-2 fuzzy sets in [11].

The following results will show that the operations of type-2 intuitionistic fuzzy sets do not have distributive laws.

Theorem 3.2.7. Let $f, g, h$ be in $\boldsymbol{M}$ We have

$$
\begin{aligned}
& 1 . f \uplus(g \cap h)=\left[f_{R} \triangle\left(g_{L} \nabla h\right)\right] \vee\left[f_{R} \triangle\left(g \nabla h_{L}\right)\right] \vee\left[f \triangle\left(g_{R L} \nabla h_{R}\right)\right] \vee \\
& {[f \triangle}\left.\left(g_{R} \nabla h_{R L}\right)\right] . \\
& 2 .(f \uplus g) \cap(f \cup h) \quad=\left[\left(f_{R L} \triangle g_{L}\right) \nabla\left(f_{R} \triangle h\right)\right] \vee\left[\left(f_{R L} \triangle g_{L}\right) \nabla\left(f \triangle h_{R}\right)\right] \\
& \vee\left[\left(f_{L} \triangle g_{R L}\right) \nabla\left(f_{R} \triangle h\right)\right] \vee\left[\left(f_{L} \triangle g_{R L}\right) \nabla\left(f \triangle h_{R}\right)\right] \\
& \vee\left[\left(f_{R} \triangle g\right) \nabla\left(f_{R L} \triangle h_{L}\right)\right] \vee\left[\left(f_{R} \triangle g\right) \nabla\left(f_{L} \triangle h_{R L}\right)\right] \\
& \vee\left[\left(f \triangle g_{R}\right) \nabla\left(f_{R L} \triangle h_{L}\right)\right] \vee\left[\left(f \triangle g_{R}\right) \nabla\left(f_{L} \triangle h_{R L}\right)\right] .
\end{aligned}
$$




\section{Proof}

1.From the left side, we have.

$$
\begin{aligned}
f \uplus(g \cap h)= & {\left[f_{R} \nabla(g \cap h)\right] \vee\left[f \nabla(g \cap h)_{R}\right] } \\
= & \left\{f_{R} \nabla\left[\left(g_{L} \triangle h\right) \vee\left(g \triangle h_{L}\right)\right]\right\} \vee\left\{f \nabla\left[\left(g_{R} \triangle h_{R L}\right) \vee\left(g_{R L} \triangle h_{R}\right)\right]\right\} \\
= & {\left[f_{R} \nabla\left(g_{L} \triangle h\right)\right] \vee\left[f_{R} \nabla\left(g \triangle h_{L}\right)\right] \vee\left[f \nabla\left(g_{R} \triangle h_{R L}\right)\right] } \\
& \vee\left[f \nabla\left(g_{R L} \triangle h_{R}\right)\right]
\end{aligned}
$$

2.We have $(f \uplus g) \cap(f \uplus h)=\left[(f \uplus g)_{L} \nabla(f \uplus h)\right] \vee\left[(f \uplus g) \nabla(f \uplus h)_{L}\right]$ the first expression, we have

$$
\begin{aligned}
(f \uplus g)_{L} \nabla(f \uplus h)= & {\left[\left(f_{R L} \triangle g_{L}\right) \vee\left(f_{L} \triangle g_{R L}\right)\right] \nabla\left[\left(f_{R} \triangle h\right) \vee\left(f \triangle h_{R}\right)\right] } \\
= & {\left[\left(f_{R L} \triangle g_{L}\right) \nabla\left(f_{R} \triangle h\right)\right] \vee\left[\left(f_{R L} \triangle g_{L}\right) \nabla\left(f \triangle h_{R}\right)\right] } \\
& \vee\left[\left(f_{L} \triangle g_{R L}\right) \nabla\left(f_{R} \triangle h\right)\right] \vee\left[\left(f_{L} \triangle g_{R L}\right) \nabla\left(f \triangle h_{R}\right)\right]
\end{aligned}
$$

Deal with the second expression, we have

$$
\begin{aligned}
(f \uplus g) \nabla(f \uplus h)_{L}= & \\
= & {\left[\left(f_{R} \triangle g\right) \nabla\left(f_{R L} \triangle h_{L}\right)\right] \vee\left[\left(f_{R} \triangle g\right) \nabla\left(f_{L} \triangle h_{R L}\right)\right] } \\
& \vee\left[\left(f \triangle g_{R}\right) \nabla\left(f_{R L} \triangle h_{L}\right)\right] \vee\left[\left(f \triangle g_{R}\right) \nabla\left(f_{L} \triangle h_{R L}\right)\right]
\end{aligned}
$$

Hence,

$$
\begin{aligned}
(f \uplus g) \cap(f \uplus h)= & {\left[\left(f_{R L} \triangle g_{L}\right) \nabla\left(f_{R} \triangle h\right)\right] \vee\left[\left(f_{R L} \triangle g_{L}\right) \nabla\left(f \triangle h_{R}\right)\right] } \\
& \vee\left[\left(f_{L} \triangle g_{R L}\right) \nabla\left(f_{R} \triangle h\right)\right] \vee\left[\left(f_{L} \triangle g_{R L}\right) \nabla\left(f \triangle h_{R}\right)\right] \\
& \vee\left[\left(f_{R} \triangle g\right) \nabla\left(f_{R L} \triangle h_{L}\right)\right] \vee\left[\left(f_{R} \triangle g\right) \nabla\left(f_{L} \triangle h_{R L}\right)\right] \\
& \vee\left[\left(f_{R} \triangle g\right) \nabla\left(f_{R L} \triangle h_{L}\right)\right] \vee\left[\left(f_{R} \triangle g\right) \nabla\left(f_{L} \triangle h_{R L}\right)\right] \\
& \vee\left[\left(f_{R} \triangle g\right) \nabla\left(f_{R L} \triangle h_{L}\right)\right] \vee\left[\left(f_{R} \triangle g\right) \nabla\left(f_{L} \triangle h_{R L}\right)\right] \\
& \vee\left[\left(f \triangle g_{R}\right) \nabla\left(f_{R L} \triangle h_{L}\right)\right] \vee\left[\left(f \triangle g_{R}\right) \nabla\left(f_{L} \triangle h_{R L}\right)\right]
\end{aligned}
$$

In short, this theorem shows that the distributive laws do not hold.

\section{CONCLUSION}

In this paper, we have introduced a new concept of type-2 intuitionistic fuzzy set and their operations. It is hopefully more general and applicable than the ordinary intuitionistic fuzzy set. There would be an overwhelmingly large amount of applications on many different fields that derive from it.

\section{REFERENCES}

[1] K. Atanassov, Intuitionistic fuzzy sets, Fuzzy Sets and Systems 20 (2006) 87-96.

[2] K. Atanassov, New operations defined on intuitionistic fuzzy sets, Fuzzy Sets and Systems 61 (1994) 137-142. 
[3] B.C. Cuong, N.D. Phuoc, Fuzzy systems, neural networks and applications, Science and Technology Pub., Second Ed., 2006 (in Vietnamese).

[4] S.K. De, R. Biswas, A.R. Roy, Some operations on intuitionistic fuzzy sets, Fuzzy Sets and Systems 114 (2000) 477-484.

[5] J.M. Mendel, Uncertain rule-based fuzzy logic systems, Upper Saddle River, NJ, Prentice-Hall, 2001.

[6] J.M. Mendel, R.I. Rohn, F. Liu, Interval type-2 fuzzy logic systems made simple, IEEE Tran. On Fuzzy Systems 146 (2006) 808-821.

[7] J.M. Mendel, Advances in type-2 fuzzy sets and systems, Inform. Sci. 177 (2007) 84-110.

[8] N. Karnik, J. Mendel, Operations on type-2 fuzzy sets, Fuzzy Sets and Systems 122 (2001) 327-348.

[9] T.H. Nguyen, E. Walker, A first cours in fuzzy logic, Cnapman \& Hall/CRC, Boca Raton, FL, 2000.

[10] E.H. Ruspini, P. P. Bonissone, W. Peddrycz, Handbook of Fuzzy Computation, Inst. of Physics Pub., Bristol., 1998.

[11] C.L Walker, E.A. Walker, The algebra of fuzzy truth values, Fuzzy Sets and Systems 149 (2005) 309-347.

[12] Z. Xu, Intuitionistic preference relations and their application in group decision making, Inform. Sci. 177 (2007) 2363-2379.

[13] L.A. Zadeh, Fuzzy Sets, Information and Control, 8, 338-353, 1965.

[14] L.A. Zadeh, The concept of a linguistic variable and its application to approximate reasoning, Inform. Sci. 8 (1975) 199-249

Received on March 5, 2012 